\title{
Produtividade de frutos de meloeiro cultivado em substrato com três soluções nutritivas
}

\author{
Jerônimo L. Andriolo; Mastrângello E. Lanzanova; Márcio Witter ${ }^{1}$ \\ UFSM-CCR-Depto. Fitotecnia, 97105-900 Santa Maria-RS; E-mail: andriolo@creta.ccr.ufsm.br
}

\begin{abstract}
RESUMO
Comparou-se o rendimento de frutos de meloeiro cultivado em substrato com três soluções nutritivas, no interior de um túnel alto de polietileno, na UFSM, RS. A semeadura foi feita em 19/07/01 em bandejas de poliestireno e 32 dias depois as mudas foram transferidas para sacolas de polietileno contendo 4,5 L de substrato comercial, no espaçamento de 1,0 m entre fileiras e 0,30 m entre sacolas. Durante o ciclo da cultura, as plantas foram conduzidas verticalmente e dois frutos foram deixados em cada planta, removendo-se os frutos excedentes duas vezes por semana. Os tratamentos constituíram-se de três soluções nutritivas, empregadas no cultivo comercial do meloeiro. No tratamento 1 (T1) foi utilizada a solução recomendada no estado de São Paulo para o cultivo dessa espécie em NFT e nos tratamentos 2 (T2) e 3 (T3) utilizaram-se as soluções recomendadas para o cultivo da mesma espécie em substrato na França e na Espanha, respectivamente. Em cada tratamento aplicou-se o volume de $1 \mathrm{~L}$ de solução para cada planta, em intervalos semanais, via fertirrigação. A área foliar foi estimada semanalmente a partir de medidas não destrutivas no período entre os 35 e 63 dias após o plantio. A produtividade de frutos determinada ao final do experimento apresentou valores mais elevados nas plantas que receberam as soluções nutritivas T2 e T3, as quais não diferiram significativamente entre si.
\end{abstract}

Palavras-chave: Cucumis melo, fertirrigação, cultivo fora do solo, área foliar.

\begin{abstract}
Fruit yield of muskmelon plants grown in substrate under three nutrient solutions

The fruit yield of muskmelon plants grown in substrate under three nutrient solutions was obtained. The experiment was conducted in a polyethylene tunnel at the Universidade Federal de Santa Maria, Brazil. Sowing was done on $19^{\text {th }}$ July 2001 , in polystyrene trays, using a commercial substrate. On 32 days after sowing, each plant was transferred to a $4.5 \mathrm{~L} \mathrm{bag}$, placed inside the tunnel using $1.0 \mathrm{~m}$ between rows and $0.3 \mathrm{~m}$ within each bag row. During the growing period, plants were trained vertically and two fruits were kept to set in each plant. Exceeding fruits were picked out twice a week. Treatments consisted of three nutrient solutions, used in the commercial production of muskmelon crops. Treatment 1 (T1) was the nutrient solution recommended for growing this crop in NFT system in São Paulo State and treatments 2 (T2) and 3 (T3) were those used in substrate in France and in Spain, respectively. In all treatments, $1 \mathrm{~L}$ of nutrient solution was supplied weekly to each plant by fertigation. From 35 to 63 days after planting, plant leaf surface was determined weekly by non destructive measurements. Fruit yield, at the end of the experiment was significantly higher on plants supplied with T2 and T3 nutrient solutions when compared with T1 treatment. However, no significant difference was found between $\mathrm{T} 2$ and $\mathrm{T} 3$ treatments.
\end{abstract}

Keywords: Cucumis melo, fertigation, soilless culture, leaf surface.

\section{(Recebido para publicação em 08 de fevereiro de 2002 e aceito em 19 de março de 2003)}

$\mathrm{O}$ meloeiro (Cucumis melo L.) é uma das cucurbitáceas mais exigentes quanto às condições de clima e solo para o crescimento e desenvolvimento da planta. São necessárias temperaturas do ar entre 20 e $30^{\circ} \mathrm{C}$, do meio radicular superiores a $15^{\circ} \mathrm{C}$ e umidade relativa do ar entre 55 e $65 \%$. O teor de nutrientes minerais disponíveis à planta deve ser elevado, com um correto balanceamento entre os mesmos. As necessidades de água são maiores nas fases iniciais de desenvolvimento da planta e o sistema radicular é altamente sensível à asfixia provocada por excesso de água (CTIFL, 1998; Brandão Filho \& Vasconcellos, 1998; Ramos, 1999). No Brasil, a cul- tura do meloeiro se concentra em algumas regiões onde as condições ambientais são mais favoráveis, como o Rio Grande do Norte, São Paulo e Paraná (Brandão Filho \& Vasconcellos, 1998). No extremo Sul do País, a produção dessa espécie é ainda pouco expressiva e ocorre em algumas microregiões, apenas nos meses mais quentes do ano.

Uma das alternativas para o cultivo do meloeiro em regiões e/ou épocas do ano em que as variáveis do ambiente aéreo e radicular são pouco favoráveis ao crescimento da planta é o emprego do cultivo protegido e fora do solo. No Brasil, podem ser encontradas na lite- ratura informações para o cultivo dessa espécie em hidroponia usando o sistema NFT (Castellane \& Araújo, 1994). Entretanto, uma das dificuldades associadas com essa técnica é o controle da temperatura do meio radicular, a qual tende a se manter em valores próximos da temperatura do ar. Enquanto o crescimento da parte aérea das plantas e, principalmente a qualidade dos frutos, respondem favoravelmente às temperaturas elevadas entre 25 e $30^{\circ} \mathrm{C}(\mathrm{FAO}$, 1990), o mesmo não ocorre com a absorção de água e nutrientes, a qual passa a decrescer quando os valores ultrapassam o limite entre 20 e $22^{\circ} \mathrm{C}$ (Cornillon, 1987; FAO, 1990). Esse

\footnotetext{
${ }^{1}$ Bolsista de Iniciação Científica da Fundação de Amparo a Pesquisa do RS, no ano de 2001.
} 
decréscimo ocorre também em condições de baixas temperaturas, quando os valores atingem limites inferiores a $15^{\circ} \mathrm{C}$ (CTIFL, 1998). Nessas condições, o cultivo em substrato é mais adequado, devido a maior inércia térmica do meio radicular quando comparado com o NFT (Marfà \& Guri, 1999). Resultados recentes de pesquisas feitas no Brasil para avaliar o cultivo do meloeiro em substrato empregando a mesma solução nutritiva recomendada para o cultivo em NFT, mostraram queda no rendimento e na qualidade dos frutos (Villela et al., 2001). A composição da solução nutritiva a ser empregada no cultivo em substrato deve ser determinada em função da concentração de absorção dos nutrientes pela planta e das interações entre os íons que a compõem (Cañadas, 1999; Lopez, 1998; Lopez \& Alonso, 1998; Alpi \& Tognoni, 1999). Essas interações podem ocorrer tanto entre os diferentes íons, como entre os íons e as partículas do substrato, implicando antagonismos e/ou imobilizações de nutrientes. No caso do meloeiro, essas interações repercutem tanto sobre o rendimento como a qualidade dos frutos, especialmente as relações entre o N, o Ca e o K (CTIFL, 1998). Existe, portanto, a necessidade de determinar critérios específicos para o manejo da nutrição mineral do meloeiro cultivado em substrato.

O presente trabalho teve como objetivo comparar o crescimento foliar e o rendimento de frutos de meloeiro cultivado em substrato, empregando duas soluções recomendadas para o cultivo em substrato, respectivamente na França (T2) e na Espanha (T3), e como testemunha a solução nutritiva atualmente recomendada para o cultivo em NFT no Brasil (T1).

\section{MATERIAL E MÉTODOS}

O experimento foi instalado no Depto. de Fitotecnia da UFSM (latitude $29^{\circ} 43^{\prime} \mathrm{S}$, longitude $53^{\circ} 42 \mathrm{~W}$ e altitude $95 \mathrm{~m}$ ), no interior de um túnel alto de $180 \mathrm{~m}^{2}$, coberto com filme de polietileno transparente de $10^{-6} \mathrm{~m}$ de espessura. Foi empregado o híbrido Hales Best Jumbo, de cultivo tradicional na região. A semeadura foi feita em 19/07/ 01, em bandejas de poliestireno com 128 células, empregando substrato comercial. Aos 32 dias após a semeadura, as mu- das com quatro folhas definitivas foram transferidas para sacolas de polietileno contendo 4,5 L de substrato comercial orgânico (Plantmax $\left.{ }^{\circledR}\right)$, com apenas uma planta por sacola $\left(3,3\right.$ plantas $\left.\mathrm{m}^{-2}\right)$. As sacolas foram dispostas no interior do túnel em fileiras, no espaçamento de 1 $\mathrm{m}$ entre fileiras e $0,30 \mathrm{~m}$ entre sacolas, e perfuradas na base de forma a assegurar a livre drenagem dos volumes de água excedentes à capacidade de retenção de água do substrato. Sobre as sacolas foi instalado um tubo gotejador para o fornecimento de água e nutrientes, ajustando-se um gotejador no centro de cada sacola. O conjunto formado pelas sacolas e o tubo gotejador foi coberto com filme de polietileno opaco de cor preta, para reduzir a evaporação superficial e evitar a incidência direta dos raios solares sobre as sacolas.

As plantas foram conduzidas verticalmente com uma haste, por meio de fitas plásticas. $\mathrm{O}$ desbaste foi feita deixando-se dois frutos por planta, localizados entre o $13^{\circ}$ e $17^{0}$ entrenó. Todas as demais ramificações laterais foram eliminadas. O túnel foi ventilado nos dias ensolarados, por meio do soerguimento das extremidades laterais do filme de polietileno em até $1 \mathrm{~m}$ de altura, entre as 9 e 18:00 h, aproximadamente.

Do plantio até o final da colheita dos frutos, os nutrientes foram fornecidos às plantas uma vez por semana, por meio da fertirrigação. Os tratamentos foram constituídos por três soluções nutritivas. A testemunha (T1) foi a solução nutritiva recomendada por Castellane \& Araújo (1994) para o cultivo do meloeiro em NFT (Tabela 1). Os tratamentos T2 e T3, pelas soluções nutritivas recomendadas para o cultivo dessa espécie em substrato na França (CTIFL, 1998) e na Espanha (Ramos, 1999), respectivamente. O ferro foi fornecido na forma quelatizada ( $5 \%$ de $\mathrm{Fe})$, na proporção de $0,13 \mathrm{~mL} \mathrm{~L}^{-1}$, e os demais micronutrientes através de $0,7 \mathrm{~mL} \mathrm{~L}^{-1}$ da solução padrão de micronutrientes proposta por Jeannequin (1987), descrita por Andriolo (1999). Foi fornecido o volume de $1 \mathrm{~L}$ de solução nutritiva por planta, em doses semanais, até o final do experimento. No período entre duas fertirrigações sucessivas, somente água foi fornecida diariamente às plantas. A freqüência das irrigações foi ajustada de acordo com a demanda evaporativa da atmosfera. Em cada irrigação, o fluxo de água foi interrompido logo aos primeiros sinais de escorrimento na parte inferior das sacolas, de forma a reduzir ao máximo as perdas de nutrientes por lixiviação.

A concentração da solução nutritiva em torno das raízes foi monitorada diariamente medindo-se a condutividade elétrica (EC) da solução drenada, em cada um dos tratamentos. Para efetuar essa medida, foi instalado um coletor de volume drenado contendo cinco plantas, no interior de uma parcela, em cada um dos tratamentos. O delineamento experimental empregado foi o inteiramente casualizado, com três repetições e 20 plantas por parcela. Cada um dos tratamentos foi aplicado a uma fileira inteira de plantas, mantendo-se as duas fileiras laterais extremas do túnel como bordaduras.

A área foliar das plantas foi determinada aos 35; 43; 50; 57 e 63 dias após o plantio, quando foi feito o desponte. Em cada tratamento, foram selecionadas três plantas, sobre as quais foram efetuadas medidas não destrutivas de comprimento (C) e largura (L) de cada uma das folhas existentes (comprimento $>2 \mathrm{~cm}$ ). Semanalmente foi coletada um planta adicional em cada uma das fileiras bordaduras. Logo após a coleta, as folhas foram removidas, a área foliar determinada pelo método não destrutivo $(\mathrm{C} \times \mathrm{L})$ e 50 discos $\left(0,5052 \mathrm{~cm}^{2}\right.$ de área $)$ foram coletados de cada folha. As folhas e os discos coletados foram submetidos à secagem em estufa de circulação de ar forçado, na temperatura de $60^{\circ} \mathrm{C}$, durante uma semana, para determinação da massa seca. Uma relação foi estabelecida entre a massa seca e a área dos discos, a qual foi empregada para estimar a área foliar a partir da massa seca de folhas de cada planta. Ao final do experimento, uma segunda relação foi ajustada entre a área foliar estimada pelos dois métodos $(\mathrm{AF}=0,99(\mathrm{C} \times \mathrm{L})$ $\left.-249,8, \mathrm{R}^{2}=0,99\right)$, a qual foi empregada para converter os resultados das medidas não destrutivas feitas nas plantas de cada tratamento, em valores equivalentes àqueles obtidos pelo método dos discos. Os frutos foram colhidos ao apresentarem sinais de rachadura em torno do pedúnculo (CTIFL, 1998) e pesados. Em cada coleta, as médias de área foliar 
foram submetidas ao teste $t$ de Student, para determinar a significância das diferenças entre os tratamentos. Os resultados referentes à massa média dos frutos e produtividade de frutos foram submetidos à análise da variância e a significância das diferenças entre as médias dos tratamentos determinada pelo teste de Duncan a 5\% de probabilidade.

\section{RESULTADOS E DISCUSSÃO}

Os valores médios semanais da EC da solução drenada foram menores em T1, seguidos por T2 e T3 (Figura 1). Os valores mais baixos foram observados ao final do experimento, nos três tratamentos, atribuídos à forte lixiviação decorrente da elevada freqüência das irrigações nesse período, que se caracterizou por altos valores de radiação solar incidente e temperatura do ar. Os valores médios da $\mathrm{EC}$ durante todo o período experimental foram de 1,7; 2,2 e 2,9 dS m $\mathrm{d}^{-1}$, respectivamente para $\mathrm{T} 1$, T2 e T3.

O crescimento da área foliar da cultura foi menor nas plantas que receberam a solução $\mathrm{T} 1$, com diferenças significativas a partir dos $43 \mathrm{DAP}(\mathrm{P}<0,5)$, até o final do experimento (Figura 2). Não foram constatadas diferenças significativas para essa variável entre T2 e T3. Situação semelhante foi observada com relação à massa média e rendimento de frutos (Tabela 2). Os valores mais elevados foram observados nas plantas que receberam a solução T3, que não diferiram de T2. As plantas que receberam a solução T1 apresentaram valores 19,1\% inferiores para essas duas variáveis.

Os valores máximos de EC da solução drenada apontados como limitantes em culturas de meloeiro em substrato situam-se em torno de 5,0 dS m-1 (Perez \& Lopez, 1998). Os valores máximos observados nas três soluções nutritivas comparadas no atual experimento situaram-se sempre abaixo desse limite, tendo sido por isso reduzidos os riscos de toxidez por salinidade associada à solução nutritiva. Por outro lado, o valor médio de EC de $1,7 \mathrm{dS} \mathrm{m}^{-1}$ da solução T1 mostrou-se limitante ao crescimento da planta e produtividade da cultura, confirmando os resultados de Amor et al. (2001), que apontam o valor de 2,0

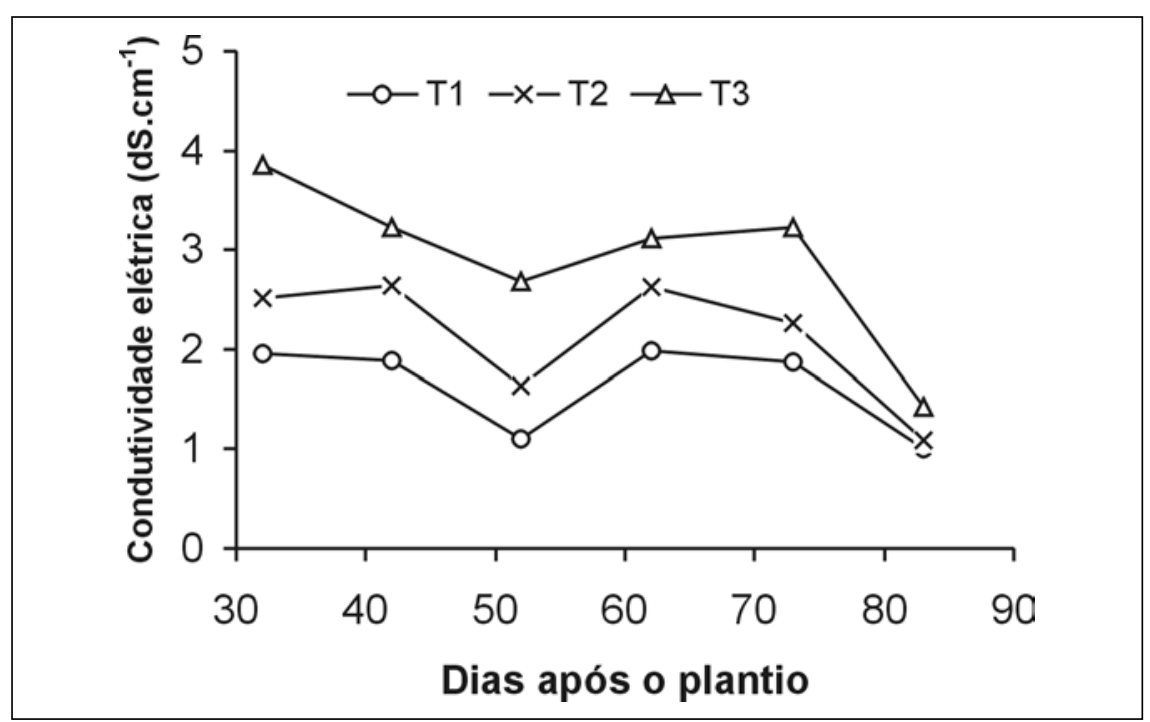

Figura 1. Evolução da condutividade elétrica da solução drenada durante o experimento, pelas três soluções nutritivas comparadas. Santa Maria, UFSM, 2001.

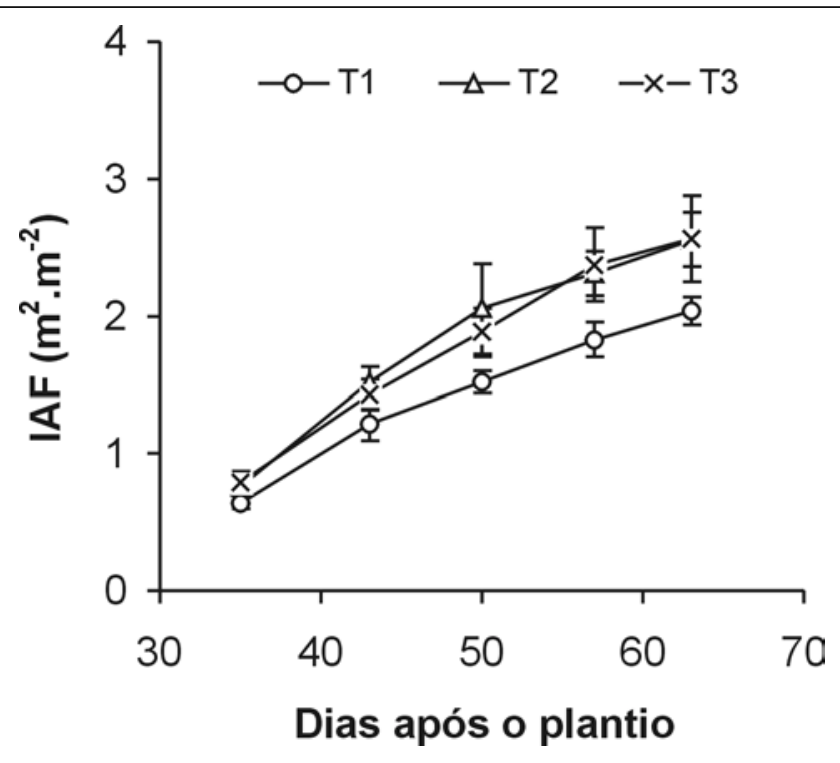

Figura 2. Evolução do índice de área foliar (IAF) de plantas de meloeiro cultivadas em substrato com três soluções nutritivas. Santa Maria, UFSM, 2001.

$\mathrm{dS} \mathrm{m}^{-1}$ como limite inferior da EC no cultivo dessa espécie em substrato.

Tanto a massa média como a produtividade de frutos de meloeiro dependem diretamente do crescimento da área foliar da planta, que deve preceder o crescimento dos frutos (CTIFL, 1998; Valantin, 1998). A acumulação e a partição da massa seca entre os órgãos vegetativos e os frutos de plantas de hortaliças cultivadas em substrato são influenciadas pela quantidade e pela proporção entre os nutrientes fornecidos pela solução nutritiva (Cortéz, 1999).
Esse princípio foi confirmado pelos atuais resultados. A concentração de íons macronutrientes fornecidos pelas três soluções empregadas, em $\mathrm{mmol} \mathrm{L}^{-1}$, foi de 24,78; 23,88 e 29,41, respectivamente em T1, T2 e T3. Isso significa que as plantas cultivadas com a solução T2 receberam uma quantidade de nutrientes $3,6 \%$ inferior àquelas cultivadas com a solução T1. Porém, mesmo recebendo através da solução T2 uma menor quantidade semanal de nutrientes, essas plantas atingiram uma produtividade de frutos $19,9 \%$ superior àquelas de $\mathrm{T} 1$. Isso 
Tabela 1. Composição das soluções nutritivas fornecidas às plantas de meloeiro cultivadas em substrato, pelos tratamentos T1, T2 e T3. Santa Maria, UFSM, 2001.

\begin{tabular}{lrcc}
\hline \multirow{2}{*}{ Nutrientes } & \multicolumn{3}{c}{ Tratamentos $\left(\mathbf{m m o l ~ L}^{-1}\right)$} \\
\cline { 2 - 4 } & $\mathbf{T} 1$ & $\mathrm{~T} 2$ & T3 \\
\hline $\mathrm{NO}_{3}^{-}$ & 12,00 & 10,71 & 13,00 \\
$\mathrm{H}_{2} \mathrm{PO}_{4}^{-}$ & 1,26 & 1,05 & 0,90 \\
$\mathrm{~K}^{+}$ & 5,68 & 4,77 & 6,00 \\
$\mathrm{Ca}^{++}$ & 3,82 & 4,71 & 5,01 \\
$\mathrm{Mg}^{++}$ & 1,01 & 1,32 & 2,25 \\
$\mathrm{SO}_{4}^{--}$ & 1,01 & 1,32 & 2,25 \\
\hline
\end{tabular}

Tabela 2. Massa média do fruto e rendimento de frutos de melão cultivado em substrato com três soluções nutritivas. Santa Maria, UFSM, 2001.

\begin{tabular}{|c|c|c|}
\hline Tratamentos & Massa média do fruto $(\mathrm{g})$ & Rendimento (t.ha- ${ }^{-1}$ ) \\
\hline T1 & $0,715 \mathrm{~b}$ & $47,15 \mathrm{~b}$ \\
\hline T2 & $0,857 a$ & $56,55 a$ \\
\hline T3 & $0,884 a$ & $58,35 a$ \\
\hline
\end{tabular}

*/Médias seguidas de mesma letra nas colunas não diferem entre si pelo teste de Duncan a $5 \%$ de probabilidade.

pode ser atribuído à maior proporção dos íons $\mathrm{K}^{+}, \mathrm{Ca}^{++}$e $\mathrm{Mg}^{++}$na composição da solução T2. Essas observações sugerem que os resultados obtidos por Villela et al. (2001) em NFT se devem provavelmente aos baixos teores de cátions da solução nutritiva empregada naquele experimento.

A qualidade dos frutos de melão é favorecida por condições de disponibilidade hídrica controlada nas fases finais do seu crescimento e maturação. Uma das formas de restringir a absorção de água pela planta é a elevação da concentração salina em torno das raízes. Por esse motivo, são recomendados valores de EC mais elevados nessas fases, próximos ao limite superior de 5,0 dS m ${ }^{-1}$ (Ramos, 1999). No atual experimento, os valores de EC nos três tratamentos mostraram decréscimo do início para o final do experimento, indicando que o manejo da freqüência das fertirrigações e/ou da concentração da solução nutritiva nesses períodos deve ser melhor ajustado à demanda hídrica da cultura, para não influenciar negativamente a qualidade dos frutos.

Os resultados obtidos indicaram que a solução nutritiva T1 é menos adequada que as soluções T2 e T3, para o cultivo do meloeiro em substrato empregando materiais orgânicos com características semelhantes àquelas do atual experimento.
Embora a produtividade de frutos não tenha diferido significativamente entre as plantas que receberam essas duas últimas soluções nutritivas, não podem ser descartadas possíveis influências da composição da solução nutritiva e/ou da condutividade elétrica sobre características visuais e/ou qualitativas dos frutos. Por esse motivo, a opção por uma ou outra dessas duas soluções deve ser fundamentada por critérios econômicos, levando-se em conta a relação custo/benefício.

\section{LITERATURA CITADA}

ALPI, A.; TOGNONI, F. Cultivo en invernadero. Madrid: Mundi-Prensa, 3 ed. 1999. 347 p.

AMOR, F.M.; FLORES, P.; CARVAJAL, M.; MARTÍNEZ, J.M.; NAVARRO, J.M.; CERDÁ, A. Yield responses of soilless melon and tomato to different irrigation water qualities. Acta Horticulturae, v. 559, p. 333-338, 2001.

ANDRIOLO, J.L. Fisiologia das culturas protegidas. Santa Maria, Editora da UFSM, 1999. 142 p. BRANDÃO FILHO, J.U.T.; VASCONCELLOS, M.A.S. A cultura do meloeiro. In: GOTO, R.; TIVELLI, S.W. (eds.). Produção de hortaliças em ambiente protegido: condições subtropicais. São Paulo: Fundação Editora da UNESP, 1998. p. 161-193. CAÑADAS, J.J.M. Sistemas de cultivo en substrato: a solución perdida y com recirculación del lixiviado. In: MILAGROS, M.F.; GÓMEZ, I.M.C. (ed.). Cultivos sin suelo II. Curso Superior de Especialización. Almería: DGIFA-FIAPACaja Rural de Almería. 1999, 2ed. p. 173-205.
CASTELLANE, P.D.; ARAÚJO, J.A.C. de. Cultivo sem solo: hidroponia. Jaboticabal: Funep, 1994. $43 \mathrm{p}$.

CORNILLON, P. Influence de la température des racines sur la croissance et la nutrition des plantes. In: BLANC, D. (ed.). Les cultures hors sol. Paris: INRA, 1987. p. 221-234.

CORTÉS, E.M. Características del riego en cultivos sin suelo: exigencias en aportación y manejo. Resultados experimentales en cultivo de pepino en perlita. In: Cultivos sin suelo II. Curso superior de especialización. Almeria: Dirección general de Investigación y Formación Agraria de la Junta de Andalucia, FIAPA, Caja Rural de Almería, 1999. p. 287-305.

CTIFL. Centre Technique Interprofessionel des Fruits e des Légumes. Le melon. Paris: CTIFL, 1998. $165 \mathrm{p}$.

FAO. Soilless culture for horticultural crop production. Rome: Food and Agriculture Organization of the United Nations, 1990. $188 \mathrm{p}$. (FAO Plant Production and Protection Paper, 101). LOPEZ, C.C. Fertirrigación: aspectos básicos. In: LOPEZ, C.C. ed. Fertirrigación. Cultivos horticolas y ornamentales. Madrid: Mundi-Prensa. 1998 , p. 65-79.

LOPEZ, C.C.; ALONSO, E.E. Calculo y preparación de disoluciones fertilizantes. In: LOPEZ, C.C. ed. Fertirrigación. Cultivos horticolas y ornamentales. Madrid: Mundi-Prensa. 1998, p. 125-172.

MARFÀ, O.; GURI, S. Física de sustratos y oxigenación del medio radicular. In: MILAGROS, M.F.; GÓMEZ, I.M.C. ed. Cultivos sin suelo II. Curso Superior de Especialización. Almería: DGIFA-FIAPA-Caja Rural de Almería. 1999, 2ed. p. 93-106.

PEREZ, M.L.S.; LOPEZ, C.C. Fertirrigación de cultivos hortícolas. In: LOPEZ, C.C. (ed.). Fertirrigación. Cultivos hortícolas yornamentales. Madrid: Mundi-Prensa. 1998, p. 374-389.

RAMOS, J.M.C. El cultivo del melón en hidroponia. In: MILAGROS, M.F.; GÓMEZ, I.M.C. (eds.). Cultivos sin suelo II. Curso Superior de Especialización. Almería: DGIFA-FIAPACaja Rural de Almería. 1999, 2ed. p. 535-561.

VALANTIN, M. Fécondation, environnement climatique, équilibre source-puits et qualité du melon cantaloup charentais (Cucumis melo L.). Marseille: Université Aix-Marseille III, 1998. 170 p. (Thése doctorat).

VILLELA JR, L.E.V.; ARAÚJO, J.A.C.; FACTOR, T.L.; VILLELA, L.G.V. Qualidade do melão produzido em condições hidropônicas em sistemas aberto (com substrato) e fechado (tipo NFT). Horticultura Brasileira, Brasília, v. 19, suplemento CD-ROM, 2001. 